\title{
Multi-tasking CRISPR RNA scaffolds
}

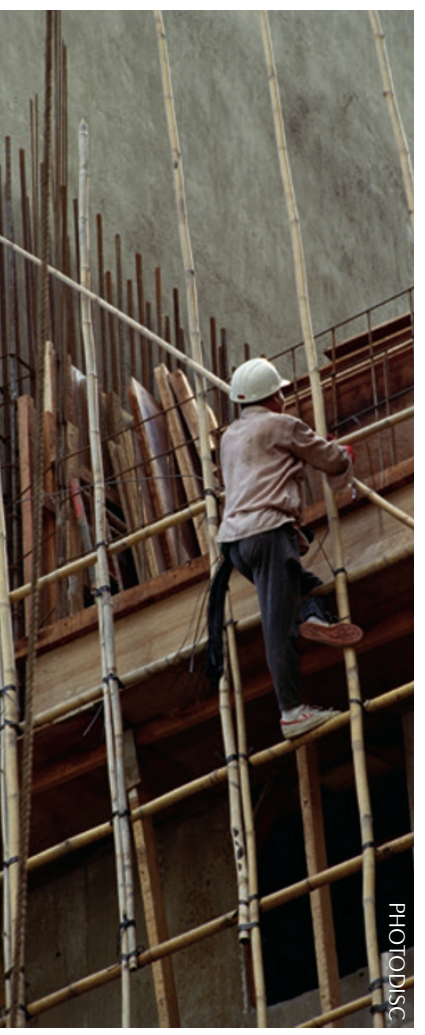

The ability to engineer or reprogramme complex gene expression networks would provide a powerful tool to not only elucidate the mechanisms underlying specific cell functions or differentiation into diverse cell types but also enable the design of cells for therapeutic or biotechnological applications. A new study reports an extension of the CRISPR (clustered regularly interspaced short palindromic repeat) technology that enables the simultaneous, tunable and directional control of expression of multiple genes. At its most basic, this method allows switching on one set of genes while switching off another set to generate synthetic multigene transcriptional programmes that can be induced to, for example, 'rewire' cell fates or engineer metabolic pathways.

To achieve flexible locus-specific transcriptional programming at multiple genes simultaneously, the researchers took advantage of features inherent to RNA, such as its modularity and programmability: RNA has previously been shown to form scaffolds that link DNA binding through base pairing to protein recruitment and the assembly of functional protein complexes.

In Saccharomyces cerevisiae, Zalatan et al. repurposed the CRISPR single-guide RNA (sgRNA) by extending its sequence with modular RNA domains that recruit RNA-binding proteins, ultimately transforming the sgRNA into a 'scaffold RNA' with additional protein recruitment capabilities. Three RNAbinding recruitment modules were tested, each of which led to a marked increase in reporter gene expression through the recruitment of their respective RNA-binding proteins fused to VP64, a transcriptional activator. This finding indicates that information on which locus to target and what regulatory function to execute can be successfully combined in a single RNA molecule.

The efficacy of scaffold RNAbased protein recruitment was confirmed in human HEK293T cells.
Further analyses in human cells also showed that the system can be used to recruit gene-silencing chromatin modifiers, and that simultaneous targeting of two endogenous genes to achieve contrary expression (that is, the parallel repression of one gene and activation of another) is feasible.

Finally, the investigators tested the ability of programmed scaffolding RNAs to redirect metabolic pathway outputs using the bacterial violacein biosynthetic pathway, which can be reconstituted in yeast. They successfully reprogrammed the output of this branched metabolic pathway using a three-gene scaffold RNA CRISPR on-off switch.

The ability to regulate different genes in a multidirectional manner as part of the same regulatory programme has implications for a wide range of biomedical and biotechnological uses. Future studies will need to address the suitability of CRISPR scaffold RNAs for these applications.

Linda Koch

ORIGINAL RESEARCH PAPER Zalatan, J. G. et al.

Engineering complex synthetic transcriptional programs with CRISPR RNA scaffolds. Cell http:// dx.doi.org/10.1016/j.cell.2014.11.052 (2014) 